\title{
Vascular stromal mass: A unique histological entity mimics malignancy on breast magnetic resonance imaging
}

\author{
Jon Gerry, James Kuo, Kristin Jensen, Irene Wapnir
}

\begin{abstract}
Introduction: Breast magnetic resonance imaging (MRI) is gaining routine acceptance for screening of high-risk patients and is used selectively in the diagnostic work-up of women with undetermined lesions or breast cancer. Malignant masses can be differentiated from benign lesions based on margin characteristics, dynamic enhancement features and size. Case Report: A 58-year-old female underwent an excisional biopsy for a non-palpable, MRIdetected mass lesion with irregular borders. The lesion demonstrated marked enhancement on postcontrast images with rapid wash-in and plateau delay phase kinetics. After excision, a 1.5-cm vascular stromal mass (VSM) comprised of admixed fibrous and fatty tissue with a nonorganized proliferation of ectatic blood vessels lined by a single layer of cytologically bland endothelial cells was identified. The described pathological entity is histologically distinct from pseudoangiomatous stromal hyperplasia hemangioma or angiolipoma. The MRI enhancement features of the VSM mimicked those of carcinoma, attributable to the characteristic ectatic blood vessels. Moreover, the admixed fibro-fatty stroma comprising the
\end{abstract}

Jon Gerry ${ }^{1}$, James Kuo ${ }^{2}$, Kristin Jensen ${ }^{3}$, Irene Wapnir ${ }^{1}$ Affiliations: ${ }^{1} \mathrm{MD}$, Department of Surgery, Stanford Hospital and Clinics, Stanford, CA, USA; ${ }^{2} \mathrm{MD}$, Department of Radiology, Stanford Hospital and Clinics, Stanford, CA, USA; ${ }^{3 M D}$, Department of Pathology, Stanford Hospital and Clinics, Stanford, CA, USA

Corresponding Author: Jon M. Gerry, MD, Department of Surgery, 300 Pasteur Drive, H-3691, Stanford University School of Medicine, Stanford, California 94305-5655. USA; 1-480-861-4836; Email: jgerry@stanford.edu

Received: 10 December 2013

Accepted: 14 February 2013

Published: 01 August 2013 mass is otherwise radiographically and sonographically similar to adjacent breast tissue, making it undetectable by mammography and ultrasound. Conclusion: The VSM is a unique histological entity visualized only by breast MRI. Its appearance is indistinguishable from malignant lesions on MRI and so requires a biopsy.

Keywords: Vascular stromal mass (VSM), Pseudoangiomatous stromal hyperplasia (PASH), Hemangioma, Angiolipoma

$$
* * * * * * * * *
$$

Gerry J, Kuo J, Jensen K, Wapnir I. Vascular stromal mass: A unique histological entity mimics malignancy on breast magnetic resonance imaging. International Journal of Case Reports and Images 2013;4(8):436-439.

$$
* * * * * * * * *
$$

doi:10.5348/ijcri-2013-08-350-CR-9

\section{INTRODUCTION}

Breast magnetic resonance imaging (MRI) is gaining acceptance for use in screening of patients at high-risk of developing breast cancer and as an additional diagnostic study in the work-up of women with undetermined breast lesions or breast cancer. Mammography is the only prospectively tested breast cancer screening imaging modality that has been validated in the detection of breast cancer. It is also uniquely effective in the discovery of microcalcifications associated with carcinoma in situ. However, it has limited sensitivity in women with dense breasts [1] Ultrasound has been incorporated more regularly into the routine evaluation of patients with breast complaints, largely used to characterize lesions 
discovered on physical exam or another imaging modality. It can differentiate solid from cystic lesions as well as define the margins of breast masses. Dedicated breast MRI was introduced in 1990s. It employs a gadolinium-based contrast agent and its detection of lesions is dependent on the neovasculature or existing capillaries within lesions [2]. In a retrospective analysis of women 25-89 years of age, dedicated breast MRI had a $92 \%$ sensitivity and an $89 \%$ specificity when used either as a screening or diagnostic tool [3]. The falsepositive rate was $11 \%$, and most false-positive specimens were described as 'benign breast tissue'. While non-mass lesions tend to account for a majority of false-positive findings on MRI, malignant mass lesions can be differentiated from benign ones based on margin characteristics, dynamic enhancement features, and size [4]. When breast MRI is used in combination with mammography and breast ultrasound, the excisional biopsy rate may double in exchange for a $10 \%$ increase in detection of invasive cancer [5].

\section{CASE REPORT}

A 58-year-old female was referred from another facility because of an MRI-detected mass in the right breast. The study was prompted after routine screening mammography detected an area of focal asymmetry. Additional diagnostic work-up showed this area to be overlapping glandular tissue without a corresponding sonographic abnormality. The patient had a significant family history for a mother diagnosed with breast cancer in her fifties, which led her to seek a second opinion. Her past medical history was unremarkable aside from early onset menopause at age 30 following a total abdominal hysterectomy and bilateral salpingooophorectomy for benign disease. She, subsequently, took hormone replacement therapy for 28 years.

On physical exam, the breasts were large and pendulous with a normal contour. Repeat diagnostic mammography and ultrasound at our institution revealed a focal asymmetry with corresponding acoustic shadowing in the right breast. A core needle biopsy was taken of the abnormality under ultrasound guidance and a marker was placed. This proved to be benign breast tissue. A repeat breast MRI was performed with a 1.5 $\mathrm{T}$ scanner. On precontrast images, the mass was both $\mathrm{T} 1$ and $\mathrm{T} 2$ isointense to the surrounding breast parenchyma. The mass demonstrated marked enhancement on postcontrast images with rapid washin and plateau delay phase kinetics with irregular margins (Figure 1A-D). The location of the biopsy marker was discordant, specifically $3.5 \mathrm{~cm}$ anterior and lateral to the $2.5 \times 1.7 \times 2.4 \mathrm{~cm}$ contrast-enhancing mass lesion.

An MRI-guided wire localization with excisional biopsy was performed because of the suspicious characteristics of this mass lesion and the discordant radiopathological findings on core needle biopsy. A $6.0 \times 5.5 \times 2.5 \mathrm{~cm}$ specimen was excised, margins were inked, and the tissue was serially sliced and radiographed after fixation overnight. No gross or specimen radiograph abnormalities were identified. Microscopic analysis showed proliferative fibrocystic changes. A 1.5-cm mass-like lesion comprised admixed fibrous and fatty tissue was focally identified. Within this area these was a non-organized proliferation of mildly to moderately ectatic blood vessels lined by a single layer of cytologically bland endothelial cells (Figure 2A-B). Immunohistochemical studies using antibodies to CD31, CD34 and calponin highlighted these cells but did not show p63 nuclear staining, suggesting that the lining of the vessels was a true endothelium. No inter-anastomosing channels, cellular atypia, or evidence of rapid cellular proliferation to suggest angiosarcoma was noted.
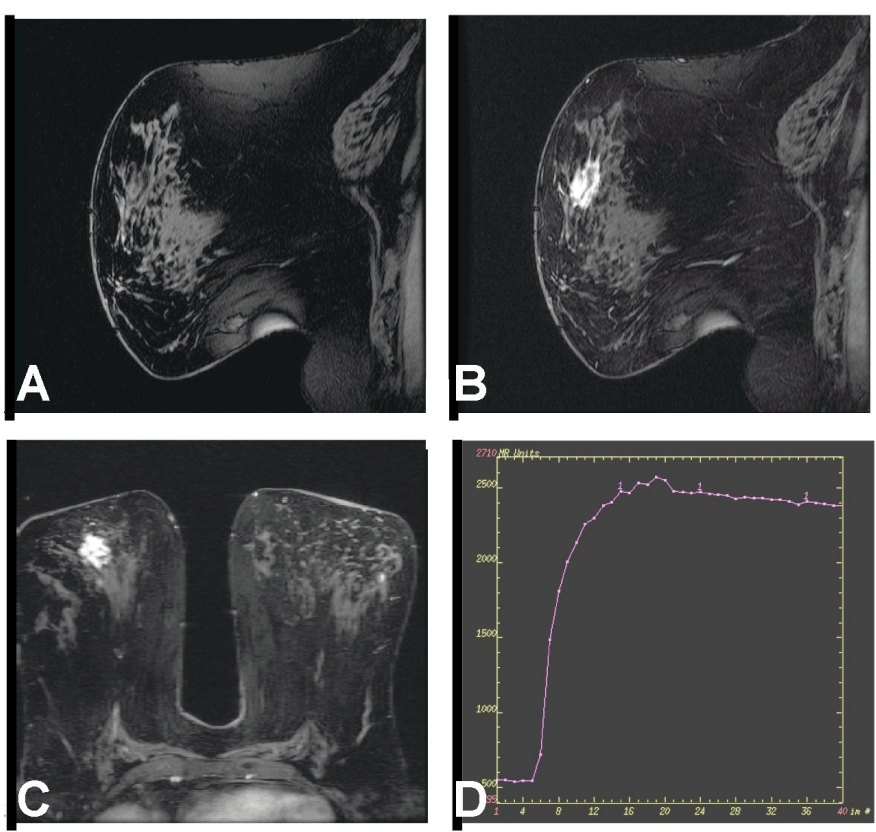

Figure 1: Mass with irregular margins in the right breast, $4.5 \mathrm{~cm}$ from nipple. (A) Precontrast sagittal T2-weighted magnetic resonance imaging of the right breast, (B) Postcontrast sagittal T2-weighted magnetic resonance imaging of the lesion in the right breast, (C) Postcontrast T2-weighted axial magnetic resonance imaging of the lesion, (D) Contrast enhancement kinetic profile showing a rapid wash-in and a plateau in the delay phase.

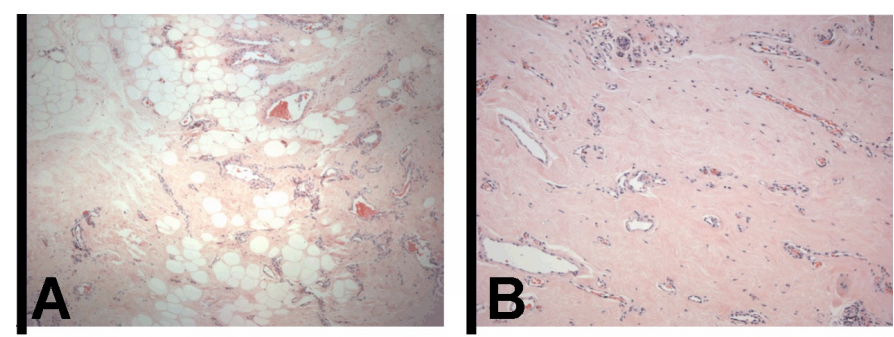

Figure 2: (A) Low-magnification image of the excised mass demonstrates vascular proliferation with admixed fibrous and fatty tissue (H\&E stain, x50), (B) Higher magnification image of the mass reveals a bland cytology of the vessels and dense stroma (H\&E stain, x100). 


\section{DISCUSSION}

Herein, we describe a non-palpable, suspicious mass identified on breast MRI, called a vascular stromal mass (VSM), which is histologically distinct from pseudoangiomatous stromal hyperplasia (PASH), hemangiomas, and angiolipomas, although these benign breast lesions bear the closest histological resemblance to the VSM. The VSM has a tortuous yet bland vasculature contained within a discrete fibro-fatty stromal mass. The PASH is characterized by a dense collagenous stroma arranged around anastomosing cleft spaces lined with spindle-shaped myofibroblasts and not actual vascular endothelium [6]. In contrast, the VSM consists of endothelium-lined ectatic blood vessels that were confirmed on immunohistochemical stains. Hemangiomas tend to contain capillary or cavernous vascular components with bland-appearing endothelial cells, and they are divided into lobules by fibrous septations [7]. The VSM vascular component is not organized into lobules. Also, the VSM has a more prominent stromal component than a hemangioma. Hemangiomas may also contain tiny calcified phleboliths, presumably caused by hemostasis within the hemangioma and thrombus formation. Phleboliths were not observed in the VSM. Angiolipomas are extremely rare in the breast. They are characterized by a predominance of adipocytes and the presence of hyaline thrombi [8]. The VSM has a predominant fibrous stromal component and there were no hyaline thrombi noted within the mass.

An MRI characteristics of the VSM are different from PASH, hemangiomas and angiolipomas, but indistinguishable from carcinoma based on margin characteristics and contrast enhancement kinetics. Importantly, the VSM is T1 and T2 isointense and is only visualized on MRI after administration of intravenous contrast with rapid wash-in and plateau delay phase kinetics. The PASH lesions demonstrate variable MRI signal intensity with a slower wash-in that continues during the delay phase [9]. Breast hemangiomas are T2 hyperintense and have increased contrast enhancement on the delay phase similar to PASH [10]. Angiolipomas of the breast are rare, and MRI characteristics have not been reported. Angiolipomas of spine have variable T1 signal intensity, are typically $\mathrm{T} 2$ hyperintense, and display variable contrast enhancement. Finally, the irregular margins of the VSM distinguish it from PASH, hemangiomas, and angiolipomas.

\section{CONCLUSION}

We characterize vascular stromal mass as a new benign histological entity which exhibits features on breast magnetic resonance imaging reminiscent of malignant lesions. If discovered on a core needle biopsy, complete surgical excision would be advised given its rarity.

\section{Author Contributions}

Jon Gerry - Conception and design, Acquisition of data, Analysis and interpretation of data, Drafting the article, Critical revision of the article, Final approval of the version to be published

James Kuo - Conception and design, Acquisition of data, Analysis and interpretation of data, Drafting the article, Critical revision of the article

Kristin Jensen - Conception and design, Acquisition of data, Analysis and interpretation of data, Drafting the article, Critical revision of the article

Irene Wapnir - Conception and design, Acquisition of data, Analysis and interpretation of data, Drafting the article, Critical revision of the article, Final approval of the version to be published

\section{Guarantor}

The corresponding author is the guarantor of submission.

\section{Conflict of Interest}

Authors declare no conflict of interest.

\section{Copyright}

(C) Jon Gerry et al. 2013; This article is distributed under the terms of Creative Commons attribution 3.0 License which permits unrestricted use, distribution and reproduction in any means provided the original authors and original publisher are properly credited. (Please see www.ijcasereportsandimages.com /copyright-policy.php for more information.)

\section{REFERENCES}

1. Hooley RJ, Andrejeva L, Scoutt LM. Breast cancer screening and problem solving using mammography, ultrasound, and magnetic resonance imaging. Ultrasound Quarterly 2011;27(1):23-47.

2. Morris E, Port E. Breast magnetic resonance imaging. In: Kuerer $\mathrm{H}$, editor. Kuerer's Breast Surgical Oncology. United States: McGraw-Hill 2010:409-27.

3. Hillman BJ, Harms SE, Stevens G, et al. Diagnostic performance of a dedicated 1.5-T breast MR imaging system. Radiology 2012;265(1):51-8.

4. Baltzer PA, Benndorf M, Dietzel M, Gajda M, Runnebaum IB, Kaiser WA. False-positive findings at contrast-enhanced breast MRI: a BI-RADS descriptor study. AJR Am J Roentgenol 2010;194(6):1658-63.

5. Berg WA, Zhang Z, Lehrer D, et al. Detection of breast cancer with addition of annual screening ultrasound or a single screening MRI to mammography in women with elevated breast cancer risk. JAMA 2012;307(13):1394-404.

6. Vuitch MF, Rosen PP, Erlandson RA. Pseudoangiomatous hyperplasia of mammary stroma. Hum Pathol 1986;17(2):185-91.

7. Jozefczyk MA, Rosen PP. Vascular tumors of the breast II: Perilobular hemangiomas and 
hemangiomas. Am J Surg Pathol 1985;9(7):491-503.

8. Cheung YC, Wan YL, Ng SH, Ng KK, Lee KF, Chao TC. Angiolipoma of the breast with microcalcification: mammographic, sonographic, and histological appearances. Clin Imaging 1999;23(6):353-5.

9. Solomou E, Kraniotis P, Patriarcheas G. A case of giant pseudoangiomatous stromal hyperplasia of the breast: magnetic resonance imaging findings. Rare Tumors 2012;4(2):e23.

10. Glazebrook KN, Morton MJ, Reynolds C. Vascular tumors of the breast: mammographic, sonographyic, and MRI appearances. Am J Roentgenol 2005;184(1):331-8.

Access full text article on other devices

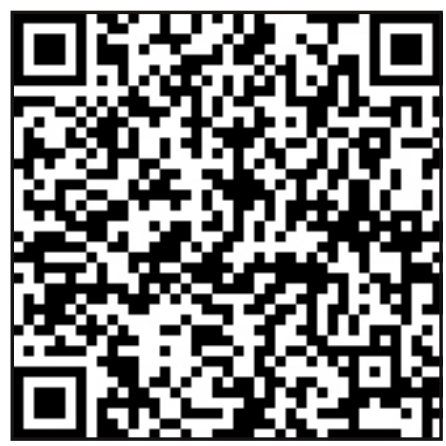

Access PDF of article on other devices

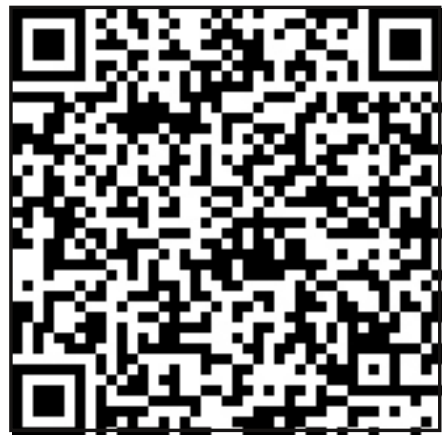

